\section{Activation analysis in vivo}

\author{
from Keith Boddy
}

The Second East Kilbride Conference on Progress and Problems of In vivo Activation Analysis, organised by the Scottish Universities Research and Reactor Centre, was held on April 6-9, 1976.

Deliberate induction of radioactivity in animal tissues was reported in Nature almost $27 \mathrm{yr}$ ago and some $12 \mathrm{yr}$ have passed since the feasibility of in vivo neutron activation analysis in man was demonstrated. The technique involves exposure of the total body or the relevant region of the body, such as the thyroid, liver, spine or limb, to a low dose of neutrons and measurement of either the induced radioactivity or the prompt gamma rays emitted during irradiation. Total body in vivo neutron activation analysis (TBIVNAA) is a unique method providing direct and simultaneous measurements of the total body content of calcium, phosphorus, nitrogen, sodium and chlorine. Similarly, there are partial body methods (PBIVNAA) for determining the total thyroid content of iodine, calcium and phosphorus in sections of bone or cadmium and copper in the liver. The reproducibility of measurements is generally $2-4 \%$, permitting the detection of relatively small changes in body composition due to disease or treatment, the patient being examined on an outpatient basis.

It was clear from the increasing number of centres using, or proposing to use, in vivo activation analysis and from the wide range of clinical applications described at the conference that the potential of the technique in medicine is being realised. Several groups are using TBIVNAA in examinations of osteoporosis, osteomalacia, renal failure, hyperparathyroidism and endocrine dysfunctions. Regional measurements of calcium in bone are being made in some of these disorders and of thyroid iodine in hyperthyroidism.

The facilities at Brookhaven National Laboratory, New York, comprising 14 sources each of $50 \mathrm{Ci} \mathrm{Pu} \mathrm{Be}$ distributed along the anterior and posterior body surfaces for irradiation and the sophisticated whole-body counter using 54 sodium iodide detectors, were described by $\mathrm{S}$. H. Cohn. A wide variety of clinical studies are in progress: in thalassaemia, for example, gross depletion of skeletal calcium $(50 \%)$ has been measured.
J. H. Fremlin (University of Birmingham) and his colleagues use a cyclotron for clinical measurements involving delayed gamma-ray emission and also for prompt gamma-ray analysis of total body nitrogen using hydrogen as an internal standard, as well as for the detection of cadium in liver. In regional analyses, spinal calcium is measured. The feasibility of determining copper and ion in liver in pathological situations has been demonstrated using PBIVNAA, and preliminary studies of their measurement by nuclear resonance fluorescence were described.

W. B. Nelp (University of Washington) and H. E. Palmer (Battelle Northwest Laboratories, Washington) and coworkers also use a cyclotron in a wide range of clinical studies. The technique of measuring total body calcium using the reaction ${ }^{40} \mathrm{Ca}(\mathrm{n}, \alpha)$ ${ }^{37} \mathrm{Ar}$, first demonstrated by Palmer, in which the exhaled gas is collected, purified and counted in proportional counters, is now in clinical use. To measure calcium alone, smaller radiation doses can be used and whole-body counting is not required. This technique has also been adopted at Birmingham and at the Memorial Sloan Kettering Cancer Center, New York (J. S. Laughlin). The rate and completeness of exhalation of ${ }^{37} \mathrm{Ar}$ has been examined in detail by several groups. Although the exhalation pattern found was obviously complex, there was preliminary evidence that the technique was reproducible and could estimate total body calcium quantitatively in man.

The Medical Research Council cyclotron at Hammersmith Hospital is used by T. Spinks and colleagues for similar studies, and in some cases they measure calcium in the hand and the rate of removal of induced ${ }^{24} \mathrm{Na}$.

In the system used at the University of Toronto by K. G. McNeil and J. E. Harrison, 12 sources each of $5 \mathrm{Ci}$ of $\mathrm{Pu}-\mathrm{Be}$ are distributed only about the thorax and lumbar region of the patient, precluding absolute measurements of total body content. But calcium and sodium indices have been derived, relating the expected countingrates of these elements to body habitus, and the measured counting rate in the patient is compared with that expected as an indicator of normality. This approach has been used in various clinical disorders. Measurement of nitrogen in vivo is also being attempted by the prompt gamma-ray method.

At East Kilbride, K. Boddy's group uses scanning irradiation with two sealed-tube 14-MeV neutron generators and a scanning shadow-shield wholebody counter for TBIVNAA. Changes of body composition in rheumatoid arthritis during treatment with non- steroid analgesics and with corticosteroids have been studied. The nuclear reactor is used to measure the thyroidal content of iodine in hyperthyroid patients, as M. J. Hooper (Western Infirmary, Glasgow) reported. A technique of "compound irradiation" with mixed neutron beams has been developed to improve the uniformity of activation in partial body calcium measurements.

A facility using two californium-252 neutron sources each of $200 \mu \mathrm{g}$ for partial body in vivo neutron activation analysis, developed by the East Kilbride group, is being used for clinical studies by M. A. Smith and colleagues at the Western General Hospital, Edinburgh. A much larger californium252 source of $3 \mathrm{mg}$ is used by A. D. LeBlanc and colleagues at Baylor College and the NASA/Johnson Space Centre, Houston, in a facility for measuring regional changes in skeletal calcium in man and whole-body measurements of small animals. A facility described by B. Mazière (CEA, Orsay), uses two sources each of 200 $\mu \mathrm{g}$ of ${ }^{252} \mathrm{Cf}$ together with four sources each of $10 \mathrm{Ci}$ of ${ }^{238} \mathrm{Pu}-\mathrm{Be}$ for the simultaneous measurement of calcium and phosphorus in the hand.

Many participants felt it was desirable to be able to compare directly the results from different centres. A proposal by Palmer to establish an international intercomparison, based on anthropomorphic phantoms containing human skeletons, to be measured "blind" by the various groups and subsequently analysed chemically, was received enthusiastically. Palmer and Boddy were appointed co-chairmen with the task of establishing the interlaboratory comparison.

\section{Influence of grazing}

\section{from Peter D. Moore}

SOME controversy surrounds the degree of influence exerted by man's grazing animals on the vegetation of the arid parts of the world. There is much room for argument and speculation simply because there is a paucity of factual information, particularly regarding the history of vegetation development. The processes at work have taken considerable periods of time-many millennia in the case of the Middle East-and the separation of climatic and anthropogenic influences often proves impossible in attempts to assess the underlying factors which have determined the structure and composition of extant vegetation. Some effort has recently been made to approach the 